\title{
Datenschutzrechliche Bedenken beim Einsatz von RFID-Technologie aus europäischer Perspektive ${ }^{1}$
}

\author{
Ulrike Verch
}

Im europaweiten Vergleich gehören die Niederlande und Finnland zu den Ländern, in denen die RFID-Technologie (radio-frequency identification) am weitesten verbreitet ist. Im Jahr 2009 nutzten nach einer Erhebung von EuroStat, dem Statistischen Amt der Europäischen Union (EU), 9 \% der niederländischen Unternehmen RFID-Chips, hauptsächlich zur Produkt- und Personenidentifizierung sowie zur Zugangskontrolle. Der entsprechende Vergleichswert für die Bundesrepublik Deutschland liegt bei $4 \%$ und der europäische Durchschnittswert bei $3 \% .^{2}$ Im Jahr 2008 wurden weltweit über 2 Mrd. RFID-Etiketten, wie sie in der Logistik, im Gesundheitsbereich oder auch in Reisepässen und Bibliotheken Anwendung finden, verkauft, davon ungefähr ein Drittel innerhalb Europas. Der Marktwert wird auf rund 4 Mrd. Euro geschätzt und die Europäische Kommission geht davon aus, dass er auf über 20 Mrd. Euro innerhalb der nächsten zehn Jahre steigen wird. ${ }^{3}$

Während die in den Jahren 1995 und 2002 erlassenen Datenschutzrichtlinien der EU ${ }^{4}$ die RFID-Technologie nicht explizit erfassen, hat sich die Europäische Kommission durch zahlreiche Veröffentlichungen und Initiativen ${ }^{5}$ in den vergangenen Jahren mehrfach des Themas angenommen und mit ihren öffentlichen Online-Konsultationen zur Bürgerbeteiligung aufgerufen. ${ }^{6}$ Die Ergebnisauswertung von 2190 Antworten der OnlineKonsultation aus dem Jahr 2006 ergab, dass innerhalb der europäischen Bevölkerung große Vorbehalte und Ängste gegen die RFID-Technologie in Hinblick auf den Schutz der Privatsphäre bestehen. So gaben $70 \%$ der Befragten an, dass sie angemessene technische Sicherheitsvorkehrungen zum Schutz ihrer Daten erwarten. Fast genauso viele Bürger wünschten sich mehr Informationen, die über die Potentiale und Risiken der neuen Technologie aufklären und 55 \% der Bevölkerung sahen einen gesetzlichen Regelungsbedarf für den Einsatz von RFID-Chips. ${ }^{7}$

1 Dieser Beitrag fasst den Diskussionsstand im Frühjahr 2010 zusammen. Entwicklungen seit diesem Zeitpunkt konnten leider nicht mehr berücksichtigt werden.

2 Pressemitteilung STAT/10/12 vom 19.01.2010: Informations- und Kommunikationstechnologien E-Commerce machte $12 \%$ des Unternehmensumsatzes im Jahr 2008 in der EU aus.

3 Pressemitteilung (IP/09/740) vom 12.05.2009: Kleine Chips mit großen Möglichkeiten: Neue EU-Empfehlungen sorgen dafür, dass die »Strichcodes des 21. Jahrhunderts« die Privatsphäre nicht verletzen.

4 Richtlinie 95/46/EG zum Schutz natürlicher Personen bei der Verarbeitung personenbezogener Daten und zum freien Datenverkehr und Richtlinie 2002/58/EG über die Verarbeitung personenbezogener Daten und den Schutz der Privatsphäre in der elektronischen Kommunikation.

5 Eine Übersicht findet sich unter: http://ec.europa.eu/information_society/policy/rfid/events/past/index_en.htm.

6 Pressemitteilung IP/06/909vom 03.07.2006:Kommission startet öffentliche Konsultation zur Funkfrequenzkennzeichnung (RFID-Technik) und siehe: http://www.rfidconsultation.eu/.

7 Mitteilung der Kommission an das Europäische Parlament, den Rat, den Europäischen Wirtschafts- und Sozialausschuss und den Ausschuss der Regionen: Funkfrequenzkennzeichnung (RFID) in Europa: Schritte zu einem ordnungspolitischen Rahmen (KOM(2007) 96. 


\section{»Your Voice on RFID«}

Begleitend zur öffentlichen Online-Konsultation konnten unterschiedliche europäische Interessens- sowie Verbraucherverbände ihre Stellungnahmen zu RFID bei der EUKommission einreichen und veröffentlichen. ${ }^{8}$ Darüber hinaus fanden in Brüssel zahlreiche Workshops und Expertenanhörungen zum Thema statt. Die Ergebnisse dieser Konsultationen wurden in einem englischsprachigen Papier mit dem Titel »Your voice on RFID « zusammengefasst. ${ }^{9}$ Neben technischen und sicherheitsrelevanten Aspekten wurden insbesondere auch datenschutzrechtliche Bedenken im Zusammenhang mit der RFID-Technologie thematisiert:

"It seems currently socially unacceptable that citizens would be tracked and traced wherever they go, all the time. If citizens buy and carry products, this could allow indirect tracking and tracing. [...] As a person can be linked to a RFID serial number (e.g., through the tag in his/her clothes), the distinction between personal data and non-personal data could be blurred.«10

Anstatt jedoch zu dieser entscheidenden Frage Stellung zu beziehen, ob eindeutig identifizierbare RFID-Chips, die Privatpersonen mit sich führen, rechtlich als personenbezogene Daten einzuordnen sind und damit dem Anwendungsbereich der EU-Datenschutzrichtlinien unterfallen, kommt das Papier zu dem Schluss, dass es noch zu früh sei, um konkrete gesetzgebende Schritte einzuleiten. Statt dessen sollten zunächst die Anwendbarkeit und Eignung bestehender Rechtsvorschriften und die weitere technologische Entwicklung abgewartet sowie die Forschungsförderung in Bezug auf die RFIDTechnologie durch die Europäische Union ausgebaut werden. ${ }^{11}$

In Hinblick auf das Recht der informationellen Selbstbestimmung wird des Weiteren ausgeführt, dass Verbraucher verstärkt Informationen und Aufklärung über die Risiken der RFID-Technologie benötigen. Nur so könnten sie selbstbestimmt entscheiden, ob sie RFID-Chips nutzen möchten. Die Bürger sollten freie Wahlmöglichkeiten haben:

»RFID should be deployed in such a way that the consumer is in control and can either choose to take advantage of the benefits of RFID usage or value the costs higher and prefer to avoid RFID functionality. At the same time, choices should be easy for consumers to exercise: information concerning RFID should be clear, conspicuous and accurate. ${ }^{12}$

8 Alle sog. "Stakeholder Papers« sind online nachzulesen unter: http://www.rfidconsultation.eu/menu/24.

9 »Your voice on RFID: Background document for public consultation on Radio Frequency Identification (RFID). Summary of five workshops. Open for discussion July-September 2006.« Online unter: http://www.rfidconsultation.eu/ docs/ficheiros/Your_voice_on_RFID.pdf

10 Eigene Übersetzung der Autorin: Derzeit erscheint es sozial nicht hinnehmbar, dass Bürger aufgespürt und verfolgt werden, wo immer sie hingehen, jederzeit. Wenn Bürger Produkte kaufen und bei sich tragen, könnte dies eine indirekte Aufspürung und Verfolgung ermöglichen. [...] Da eine Person mit einer RFID-Seriennummer in Verbindung gebracht werden kann, könnte die Unterscheidung zwischen personenbezogenen und nicht personenbezogenen Daten verschwimmen.

11 »Your voice on RFID« (siehe oben).

12 Eigene Übersetzung der Autorin: RFID soll in solcher Weise angewandt werden, dass der Verbraucher die Kontrolle hat und entscheiden kann, ob er sich entweder die Vorteile der RFID-Anwendung zunutze macht oder ob er die Risiken höher bewertet und vorzieht, die RFID-Funktionen zu meiden. Gleichzeitig sollten die Wahlmöglichkeiten für die Verbrauchen einfach auszuüben sein: Informationen über RFID sollen klar, eindeutig und genau sein. 
Als weitere Maßnahmen werden vorgeschlagen, dass Selbstverpflichtungserklärungen und Musterrichtlinien für den Einsatz der RFID-Technologie erarbeitet werden, die dann wiederum von einem RFID-Ombudsmann überwacht werden könnten. Schließlich findet sich noch die Empfehlung, in Hinblick auf neue RFID-Einsatzfelder datenschutzrechtliche Risikofolgeabschätzungen (privacy impact assessments) nach kanadischem Vorbild einzuführen.

Gestützt auf die Ergebnisse der Expertenmeinungen und der Bürgeranhörung hat die EU-Kommission im Jahr 2007 in einer offiziellen Mitteilung angekündigt, dass sie innerhalb der kommenden zwei Jahre konkrete Leitlinien und Verhaltensregeln zum Einsatz von RFID-Chips erarbeiten und Forschungsaktivitäten auf diesem Gebiet fördern werde. ${ }^{13} \mathrm{Zu}$ diesem Zweck wurde eine RFID-Sachverständigengruppe ins Leben gerufen, um unter Beteiligung von Interessensvertretern den Dialog weiterzuführen, zukünftige europäische Maßnahmen abzustimmen und eine gemeinsame RFID-Strategie zu erarbeiten. ${ }^{14}$ Die datenschutzrechtlichen Empfehlungen enden mit der Ankündigung:

»Auf dieser Basis wird die Kommission analysieren, welche zukünftigen gesetzgebenden Maßnahmen für die Einhaltung des Datenschutzes und die Wahrung der Privatsphäre erforderlich sind. « 15

\section{Empfehlungen der EU-Kommission}

Wie angekündigt hat die EU-Kommission im Mai 2009 ihre »Empfehlungen zur Wahrung der Privatsphäre und des Datenschutzes in RFID-gestützten Anwendungen « veröffentlicht. ${ }^{16}$ Das Papier enthält konkrete Vorschläge zu sechs verschiedenen Handlungsfeldern. ${ }^{17}$ Neben angekündigten Maßnahmen zur Informationssicherheit und RFID-Anwendungen im Einzelhandel sind für öffentliche Einrichtungen insbesondere die Planungen zur Informationsverbesserung und zur erhöhten Transparenz interessant. So möchte die EU-Kommission ein europaweit einheitliches Kennzeichen für RFID-Anwendungen entwickeln, das den Betreiber und eine Anlaufstelle benennt, bei der weitere Angaben u. a. über den Betreiber sowie den Zweck und Umfang der Datenspeicherung erhältlich sind. $\mathrm{Zu}$ den notwendigen Informationen, die für die Bürger einfach und klar verständlich sein sollten, zählt die EU-Kommission auch die

13 »Ausgehend von den Ergebnissen der europaweiten Konsultation wird die Kommission überdies die Weiterentwicklung der Technologien für einen besseren Schutz der Privatsphäre als Mittel zur Minderung der Datenschutzrisiken unterstützen.«

14 2007/467/EG: Beschluss der Kommission vom 28. Juni 2007 zur Einsetzung der Sachverständigengruppe für Funkfrequenzkennzeichnung (RFID), in: Amtsblatt Nr. L 176 vom 06/07/2007 S. 0025 - 0030.

$15 \mathrm{KOM} / 2007 / 0096$ endg.: Mitteilung der Kommission an das Europäische Parlament, den Rat, den Europäischen Wirtschafts- und Sozialausschuss und den Ausschuss der Regionen - Funkfrequenzkennzeichnung (RFID) in Europa: Schritte zu einem ordnungspolitischen Rahmen \{SEK(2007) 312\}.

16 Empfehlungen der Kommission vom 12. Mai 2009 (2009/387/EG) zur Umsetzung der Grundsätze der Wahrung der Privatsphäre und des Datenschutzes in RFID-gestützten Anwendungen. - In: Amtsblatt Nr. L 122 vom 16/05/2009, S. 0047-0051.

17 1. Datenschutzfolgenabschätzung, 2. Informationssicherheit, 3. Informationen und Transparenz in Bezug auf die RFID-Nutzung, 4. RFID-Anwendungen im Einzelhandel, 5. Sensibilisierungsmaßnahmen, und 6. Forschung und Entwicklung. 
sog. Datenschutzfolgeabschätzung, die den Bürger über die Risiken der konkreten RFIDAnwendung aufklärt sowie über Maßnahmen, die er treffen könnte, um diese Risiken zu mindern.

»Die Ausführlichkeit der Folgeabschätzung sollte den möglichen Datenschutzrisiken, die mit der Anwendung verbunden sind, angemessen sein.«

Jeder Betreiber von RFID-Anwendungen soll nach Auffassung der EU-Kommission zukünftig eine solche Datenschutzfolgeabschätzung vornehmen, die sechs Wochen vor Einführung der Funketiketten der zuständigen nationalen Behörde vorzulegen und in der auch eine technisch und organisatorisch verantwortliche Person zu benennen ist. Ferner werden in dem Papier weitere Sensibilisierungs- und Forschungsmaßnahmen dargelegt und schließlich die EU-Mitgliedstaaten aufgefordert, innerhalb von zwei Jahren aktiv zu werden, um den Kommissionsempfehlungen nachzukommen.

In der Begründung dieser Maßnahmen stellt die EU-Kommission klar, dass nicht nur RFID-Anwendungen betroffen sind, die personenbezogene Daten verarbeiten, sondern auch solche, die lediglich eine indirekte Personenüberwachung ermöglichen, z. B. Gegenstände im Besitz von Personen, die RFID-Tags mit individueller Artikelkennzeichnung aufweisen.

»Da die RFID-Technik potenziell sowohl allgegenwärtig als auch praktisch unsichtbar ist, muss bei ihrer Einführung den Fragen der Privatsphäre und des Datenschutzes besondere Beachtung geschenkt werden.[...] Die Mitgliedstaaten und alle Beteiligten sollten insbesondere in dieser Anfangsphase der RFID-Einführung weitere Anstrengungen unternehmen, um sicherzustellen, dass RFID-Anwendungen überwacht und die Rechte und Freiheiten des Einzelnen geachtet werden.«

\section{Internet der Dinge}

Mit der Veröffentlichung der Kommissionsempfehlungen haben die politischen Debatten über RFID auf europäischer Ebene jedoch noch keinen Abschluss gefunden. Unter dem Stichwort »Internet der Dinge « (»Internet of Things « =IoT) diskutiert die Europäische Kommission weiter über mögliche gesetzliche Maßnahmen in Hinblick auf die allgegenwärtige Datenverarbeitung, die in den kommenden Jahren voraussichtlich wachsenden Einfluss auf das wirtschaftliche und gesellschaftliche Leben in Europa nehmen wird.

»Die Aussichten für diese neue Entwicklung des Internets sind so grenzenlos wie die Zahl der Gegenstände unseres Alltagslebens. Allerdings müssen wir sicherstellen, dass die Europäer als Bürger, Unternehmer und Verbraucher die Technologie gestalten und nicht umgekehrt. « ${ }^{18}$

18 Pressemitteilung (IP/09/952) vom 18.6.2009: Wenn Ihre Joghurtbecher mit Ihnen sprechen: Europa bereitet sich auf die Internet-Revolution vor. 
Als sog. Internet der Dinge, das auch unter dem Stichwort Ubiquitous Computing oder Ambient Intelligence diskutiert wird, versteht die Europäische Union die zunehmende elektronische Vernetzung von Alltagsgegenständen und anderen Objekten, die sich entweder mit dem Internet oder untereinander verbinden. Der Einsatz von RFID-Technologie wird als Teilaspekt dieser Entwicklung gesehen, von der sich die EU-Kommission sowohl wirtschaftlichen Fortschritt als auch eine verbesserte Lebensqualität für die europäischen Bürger verspricht. ${ }^{19}$ Die EU-Kommission geht von einer rasanten technologischen Entwicklung aus, die einerseits beinhaltet, dass die Größe der Tags stetig abnimmt, während andererseits die Anzahl vernetzter Gegenstände stetig ansteigt, und erwartet eine Zunahme vernetzter Alltagsgegenstände um den Faktor 100 bis 1000 in den nächsten 5 bis 15 Jahren. ${ }^{20}$ Angesichts des erheblichen Potentials dieses Wachstumsprozesses in wirtschaftlicher und sozialer Hinsicht hält es die EU für unabdingbar, die Entwicklung nicht allein der Macht des privaten Sektors zu überlassen, sondern maßgeblichen Einfluss und Kontrolle durch die öffentliche Hand auszuüben. Zu diesem Zweck hat sie im Herbst 2008 eine weitere öffentliche Online-Konsultation durchgeführt ${ }^{21}$ und im Anschluss dazu im Juni vergangenen Jahres einen Aktionsplan (»Internet of Things - An action plan for Europe«) veröffentlicht, in dem sie weitere Schritte und Maßnahmen ankündigt, wie sie die zukünftige Entwicklung des Internets der Dinge gestalten möchte. ${ }^{22}$ Umrahmt von allgemeinen politischen Ausführungen werden in diesem Aktionsplan insgesamt 14 konkrete Handlungsfelder benannt, in denen die EU-Kommission Tätigkeitsbedarf sieht. Neben Ankündigungen zu Umweltaspekten, Forschungsaktivitäten, Standardisierungsbemühungen, statistischen Auswertungen und einem verstärkten internationalen Dialog, finden sich auch Aktionsbereiche in Bezug auf RFID und Datenschutz. Zum einen schlägt die EU-Kommission vor, in Zusammenarbeit mit der Europäischen Agentur für Netz- und Informationssicherheit ENISA ${ }^{23}$ sicherheitstechnische Maßnahmen zu verfolgen, um die Privatsphäre der Bürger schon bei der Entwicklung technischer Komponenten durch eingebaute Sicherheitsmechanismen ausreichend zu schützen. Auch technische Möglichkeiten, die RFID-Funktionsweisen der Systeme durch die Nutzer individuell zu steuern, werden als ein wesentlicher Schritt der Vertrauensbildung und gesellschaftlichen Akzeptanz benannt. Zum Schutz vor Missbrauch und Wahrung der Privatsphäre beabsichtigt die Europäische Kommission die datenschutzrechtlichen Aspekte des Internets der Dinge durchgängig zu überwachen, indem sie u. a. weiter den Dialog mit unterschiedlichen Interessensvertretern sucht und sich bei Bedarf zusätzliche gesetzgebende Schritte vorbehält. Und schließlich benennt die Kommission als dritten Aktionsbereich »Das Schweigen der Chips«.

\footnotetext{
19 Mitteilung der Kommission an das Europäische Parlament, den Rat, den Europäischen Wirtschafts- und Sozialausschuss und den Ausschuss der Regionen- Internet der Dinge: ein Aktionsplan für Europa (KOM/2009/0278 endg.) vom 18.06.2009.

20 IP/09/952 und KOM/2009/0278 endg. (siehe oben).

21 Siehe: http://ec.europa.eu/information_society/policy/rfid/index_en.htm.

22 Mitteilung der Kommission an das Europäische Parlament, den Rat, den Europäischen Wirtschafts- und Sozialausschuss und den Ausschuss der Regionen - Internet der Dinge: ein Aktionsplan für Europa (KOM/2009/0278 endg.) vom 18.06.2009.

23 European Network and Information Security Agency ( http://www.enisa.europa.eu/ ).
} 
»The Commission will launch a debate on technical and legal aspects of the right to silence of the chips « which has been referred to under different names by different authors and expresses the idea that individuals should be able to disconnect from the networked environment at any time. « ${ }^{24}$

Über den Ausgang dieser Diskussion und die weiteren vorgeschlagenen Maßnahmen wird die EU-Kommission im Jahr 2012 Bericht erstatten. ${ }^{25}$ In der Zwischenzeit werden zu den genannten Aktionsbereichen wieder Expertenanhörungen, öffentliche Ausschreibungen und Online-Konsultationen stattfinden, bei denen die Bürger ihre Interessen einbringen können. ${ }^{26}$

\section{Fazit}

Die Europäische Union verspricht sich aufgrund potentieller Kostensenkungen und Effizienzsteigerungen bei den vielfältigen Einsatzmöglichkeiten von RFID-Funketiketten in unterschiedlichen gesellschaftlichen Bereichen ein großes Potential für das wirtschaftliche Wachstum in Europa. ${ }^{27}$ Um neue Geschäfts- und damit auch Beschäftigungschancen zu ermöglichen, möchte die Brüsseler Kommission den technologischen Fortschritt der Europäer bei der RFID-Technologie durch weitere Anstrengungen und Forschungsaktivitäten ausbauen und mit entsprechenden Fördermitteln unterstützen. ${ }^{28}$ Im aktuellen 7. Forschungsrahmenprogramm der Europäischen Kommission, das sich auf die Jahre 2007 bis 2013 erstreckt, ist das höchste Etatvolumen mit einer Summe von über 9 Mrd. Euro für die Erforschung neuer Informations- und Kommunikationstechnologien vorgesehen. Viele beantragte Projekte beschäftigen sich mit dem Thema RFID, sehr viele mit drahtlosen und ubiquitären Infrastrukturen und wenige Projekte mit dem Datenschutz. ${ }^{29}$ Daneben gibt es noch viele andere europäischen Fördertöpfe, aus denen sich der Ausbau der RFID-Technologie finanzieren lässt. ${ }^{30}$ So wird beispielsweise die neue Medienausleihe mittels RFID-Verbuchung in der Universitätsbibliothek der Humboldt-Universität zu Berlin durch den Europäischen Fonds für Regionale Entwicklung (EFRE) gefördert. ${ }^{31}$

Trotz dieser starken Förderaktivitäten und positiven Grundeinstellung gegenüber RFID lässt die EU-Kommission kritische Töne insbesondere hinsichtlich der Gefahren für den Datenschutz nicht verkennen:

24 Offizielle deutsche Übersetzung: »Die Kommission wird eine Debatte über die technischen und rechtlichen Aspekte des >Rechts auf das Schweigen der Chips anstoßen, auf das verschiedene Autoren unter unterschiedlichen Bezeichnungen hingewiesen haben und bei dem es darum geht, dass der Einzelne in der Lage sein sollte, sich jederzeit von seiner vernetzten Umgebung abzukoppeln.«

25 Pressemitteilung (IP/09/952) vom 18.6.2009: Wenn Ihre Joghurtbecher mit Ihnen sprechen: Europa bereitet sich auf die Internet-Revolution vor.

26 So ist im Juni 2010 in Brüssel eine Expertentagung zu dem Thema geplant, bei der eine »Roadmap for Europe« erarbeitet werden soll, siehe: http://www.eu-ems.com/summary.asp?event_id=55\&page_id=342.

27 Empfehlungen der Kommission vom 12. Mai 2009 (2009/387/EG) zur Umsetzung der Grundsätze der Wahrung der Privatsphäre und des Datenschutzes in RFID-gestützten Anwendungen. - In: Amtsblatt Nr. L 122 vom 16/05/2009, S. 0047-0051.

28 MEMO/08/145 vom 05.03.2008: Radio Frequency Identification Devices (RFID): Frequently Asked Questions.

29 Ausführliche Projektinformationen sind über die Forschungsdatenbank CORDIS abrufbar: http://cordis.europa.eu.

30 Beispielsweise das Förderprogramm CIP = Competitiveness and Innovation Framework Programme.

31 http://www.ub.hu-berlin.de/ueber-uns/partnerschaften/rfid-projekt/rfid-projekt . 
»RFID-Tags have the potential to link everyday objects into an >Internet of Things that will greatly enhance economic prosperity and the quality of life. But as with any breakthrough, there is a possible downside - in this case, the implications of RFID for privacy. This is why we need to build a society-wide consensus on the future of RFID, and the need for credible safeguards. Privacy is at the heart of our European model of society. ${ }^{32}$

Gleichwohl sind neue Datenschutzverordnungen oder Richtlinien zu RFID in Brüssel derzeit nicht geplant und auch auf bundespolitischer Ebene sind keine aktuellen Verlautbarungen über entsprechende gesetzgebende Maßnahmen erkennbar. ${ }^{33}$ Es bleibt indes die Frage offen, wie sich die Bundesrepublik Deutschland zu den Empfehlungen der EUKommission zur Wahrung der Privatsphäre in RFID-gestützen Anwendungen vom vergangenen Jahr positionieren wird. Sie ist verpflichtet, der EU-Kommission bis zum Mai 2011 mitzuteilen, welche nationalen Maßnahmen eingeleitet wurden, um den Kommissionsempfehlungen zu entsprechen. Die Europäische Union selbst will erst im Jahr 2012 über weitere mögliche Schritte und Maßnahmen entscheiden. ${ }^{34}$ Für die nahe Zukunft ist voraussichtlich eine europaweit einheitliche RFID-Kennzeichnung zu erwarten sowie die Pflicht, RFID-Beauftragte zu benennen, auch wenn sich diese Aufgabe unschwer auf die betrieblichen und behördlichen Datenschutzbeauftragten übertragen ließe. Insgesamt betrachtet werden die Informationspflichten im Zusammenhang mit dem Einsatz der Funketiketten vermutlich erheblich ausgeweitet. Eventuell sind damit auch die von der EU-Kommission geforderten Datenschutzfolgenabschätzungen verbunden.

Öffentliche Einrichtungen und Bibliotheken in Deutschland sollten sich auf eine mögliche Kennzeichnung ihrer RFID-Lesegeräte und RFID-Tags und unter Umständen umfassende Informationspflichten einstellen. Fraglich ist, ob auch eine gesetzliche Verpflichtung eingeführt wird, den Bürgern Wahlmöglichkeiten bezüglich der Nutzung von RFID-Services zu gewähren. Dies würde für Bibliotheken beispielsweise bedeuten, dass sie parallel zur RFID-Medienverbuchung alternative Ausleihmethoden vorhalten und anbieten müssten. Von Seiten der EU gibt es hierzu keine konkrete Empfehlung, jedoch wird im Zusammenhang mit dem RFID-Einsatz im Einzelhandel regelmäßig betont, dass die Verbraucher selbstbestimmt über die RFID-Nutzung entscheiden können, und die Kommission plädiert in ihren Empfehlungen für das sog. Opt-In-Modell, bei dem die RFID-Tags automatisch entfernt werden müssen, es sei denn, der Kunde widerspricht

32 MEMO/08/145 vom 05.03.2008: Radio Frequency Identification Devices (RFID): Frequently Asked Questions. Eigene Übersetzung der Autorin: RFID-Etiketten haben das Potential, Alltagsgegenstände mit dem »Internet der Dinge« zu verlinken, das den wirtschaftlichen Wohlstand und die Lebensqualität erheblich steigern wird. Gleichwohl, wie bei jedem Durchbruch, gibt es auch eine mögliche Kehrseite - in diesem Fall die Auswirkungen von RFID auf die Privatsphäre. Das ist der Grund, weshalb es notwendig ist, dass wir einen gesellschaftsweiten Konsens für die Zukunft von RFID benötigen sowie verlässliche Schutzmaßnahmen. Die Gewährleistung der Privatsphäre ist das Herz unseres europäischen Gesellschaftsmodells.

33 Die letzte ausführliche Stellungnahme der Bundesregierung zu Datenschutzaspekten bei RFID-Anwendungen datiert vom Januar 2008 und beinhaltet einen Verzicht eines gesetzgeberischen Tätigwerdens und empfiehlt die Selbstregulierung des Marktes in Form effektiver Selbstverpflichtungen; vgl. Drucksache 16/7891 vom 23.01.2008: Bericht der Bundesregierung zu den Aktivitäten, Planungen und zu einem möglichen gesetzgeberischen Handlungsbedarf in Bezug auf die datenschutzrechtlichen Auswirkungen der RFID-Technologie.

34 So zumindest ist der Zeitplan in den Empfehlungen der EU-Kommission festgehalten: Empfehlungen der Kommission vom 12. Mai 2009 (2009/387/EG) zur Umsetzung der Grundsätze der Wahrung der Privatsphäre und des Datenschutzes in RFID-gestützten Anwendungen. - In: Amtsblatt Nr. L 122 vom 16/05/2009, S. 0047-0051. 
der Deaktivierung ausdrücklich. ${ }^{35}$ Da es bei Bibliotheksbüchern im Gegensatz zum Warenkauf nicht möglich ist, die Funketiketten einfach zu entfernen und die Bibliotheken als öffentliche Einrichtungen darüber hinaus für die Bevölkerung einen wichtigen Versorgungsauftrag haben, auf den viele Bürger angewiesen sind, ließe sich das aktuell diskutierte »Recht auf das Schweigen der Chips « in Bibliotheken am effektivsten durch das Angebot alternativer Ausleihmethoden realisieren.

In mehreren Papieren der EU ist nachzulesen, dass nicht nur RFID-Anwendungen, die sich auf bestimmte oder bestimmbare Personen beziehen, eine Gefahr für die Privatsphäre darstellen, sondern auch Objekte mit einer RFID-Artikelnummer, die sich im Besitz der Bürger befinden und eine indirekte Überwachung erlauben. Für diese sog. potentiell personenbeziehbaren Daten unterliegen die Betreiber bisher nicht den strengen datenschutzrechtlichen Regelungen, obgleich die luxemburgische EU-Kommissarin für Justiz, Grundrechte und Bürgerschaft ${ }^{36}$ Viviane Reding hier durchaus einen Regelungsbedarf erkennt:

»While smart chips working with RFID technology can make businesses more efficient and better organised, I am convinced they will only be welcomed in Europe if they are used by the consumers and not on the consumers. No European should carry a chip in one of their possessions without being informed precisely what they are used for, with the choice of removing or switching it off at any time. The >Internet of Things < will only work if it is accepted by the people.« 37

35 »In addition, to guarantee consumer choice and control, RFID tags that contain personal data should be automatically deactivated at the spoint of sale<, unless the consumer decides otherwise.« Allerdings sehen die Empfehlungen auch Ausnahmen für den Fall vor, dass die zuvor durchgeführte Datenschutzfolgeabschätzung im konkreten Fall keine Gefahren für die Privatsphäre ergeben haben.

36 Im Jahr 2009 als EU-Kommissarin noch zuständig für die Informationsgesellschaft und Medien.

37 Offizielle Übersetzung: »RFID-Chips können zwar die Effizienz in Unternehmen steigern und ihre Organisation verbessern, doch ist ihre Akzeptanz in Europa nach meiner Überzeugung nur dann gewährleistet, wenn sie von den Verbrauchern und nicht gegen sie verwendet werden. Kein Europäer sollte einen Chip in seinen Sachen mit sich führen, ohne genau darüber informiert zu sein, wozu dieser verwendet wird, und ohne ihn jederzeit entfernen oder ausschalten zu können. Das >Internet der Dinge « wird nur dann funktionieren, wenn es von den Menschen akzeptiert wird« (EU-Kommissarin Reding: Datenschutz der Bürger muss im digitalen Zeitalter Priorität haben (IP/09/571) vom 14.04.2009 und MEMO/09/232. 


\section{Literatur und Internetquellen}

[1] Pressemitteilung STAT/10/12 vom 19.01.2010: Informations- und Kommunikationstechnologien E-Commerce machte $12 \%$ des Unternehmensumsatzes im Jahr 2008 in der EU aus.

[2] Pressemitteilung (IP/09/740) vom 12.05.2009: Kleine Chips mit großen Möglichkeiten: Neue EU-Empfehlungen sorgen dafür, dass die »Strichcodes des 21. Jahrhunderts« die Privatsphäre nicht verletzen.

[3] Richtlinie 95/46/EG zum Schutz natürlicher Personen bei der Verarbeitung personenbezogener Daten und zum freien Datenverkehr

[4] Richtlinie 2002/58/EG über die Verarbeitung personenbezogener Daten und den Schutz der Privatsphäre in der elektronischen Kommunikation.

[5] Pressemitteilung IP/06/909 vom 03.07.2006:Kommission startet öffentliche Konsultation zur Funkfrequenzkennzeichnung (RFID-Technik) http://www.rfidconsultation.eu/.

[7] Stakeholder Papers. http://www.rfidconsultation.eu/menu/24

[8] Your voice on RFID: Background document for public consultation on Radio Frequency Identification (RFID). Summary of five workshops. Open for discussion July-September 2006. http://www. rfidconsultation.eu/docs/ficheiros/Your_voice_on_RFID.pdf

[9] 2007/467/EG: Beschluss der Kommission vom 28. Juni 2007 zur Einsetzung der Sachverständigengruppe für Funkfrequenzkennzeichnung (RFID), In: Amtsblatt Nr. L 176 vom 06/07/2007 S. 0025-0030

[10] Empfehlungen der Kommission vom 12. Mai 2009 (2009/387/EG) zur Umsetzung der Grundsätze der Wahrung der Privatsphäre und des Datenschutzes in RFID-gestützten Anwendungen. - In: Amtsblatt Nr. L 122 vom 16/05/2009, S. 0047-0051

[11] Pressemitteilung (IP/09/952) vom 18.6.2009: Wenn Ihre Joghurtbecher mit Ihnen sprechen: Europa bereitet sich auf die Internet-Revolution vor

[12] Mitteilung der Kommission an das Europäische Parlament, den Rat, den Europäischen Wirtschaftsund Sozialausschuss und den Ausschuss der Regionen- Internet der Dinge: ein Aktionsplan für Europa (KOM/2009/0278 endg.) vom 18.06.2009

[13] European Network and Information Security Agency http://www.enisa.europa.eu/.

[14] European Commission Directorate-General Information Society and Media Network Enterprise and RFID, http://ec.europa.eu/information_society/policy/rfid/events/past/index_en.htm

[15] Universitätsbibliothek der Humboldt-Universität zu Berlin. http://www.ub.hu-berlin.de/ueber-uns/ partnerschaften/rfid-projekt/rfid-projekt

[16] Drucksache 16/7891 vom 23.01.2008: Bericht der Bundesregierung zu den Aktivitäten, Planungen und zu einem möglichen gesetzgeberischen Handlungsbedarf in Bezug auf die datenschutzrechtlichen Auswirkungen der RFID-Technologie.

Die zitierten Internetquellen wurden zuletzt am 12.08.2011 aufgerufen. 